УДК 517.55

\title{
Analytic Continuation of Power Series by Means of Interpolating the Coefficients by Meromorphic Functions
}

\author{
Aleksandr J. Mkrtchyan* \\ Institute of Mathematics and Computer Science \\ Siberian Federal University \\ Svobodny, 79, Krasnoyarsk, 660041 \\ Russia
}

Received 07.04.2015, received in revised form 20.04.2015, accepted 25.04.2015

We study the problem of analytic continuation of a power series across an open arc on the boundary of the circle of convergence. The answer is given in terms of a meromorphic function of a special form that interpolates the coefficients of the series. We find the conditions for the sum of the series to extend analytically to a neigbourhood of the arc, to a sector defined by the arc, or to the whole complex plane except some arc on the convergence disk.

Keywords: Power series, analytic continuation, interpolating meromorphic function, indicator function.

\section{Introduction}

The problem of analytic continuation and finding singular points of a function has a rich and long history. It has been studied by many prominent mathematicians such as Carlson, Polya, Hadamard, and others (see, for example, [1]). There are different approaches to studying such problems. In this paper we consider the question of continuation of a power series across the boundary of its circle of convergence. First, we recall some definitions and results.

Consider a power series

$$
f(z)=\sum_{n=0}^{\infty} f_{n} z^{n}
$$

in $z \in \mathbb{C}$, whose domain of convergence is the unit disk $D_{1}:=\{z \in \mathbb{C}:|z|<1\}$.

The Cauchy-Hadamard theorem yields that

$$
\varlimsup_{n \rightarrow \infty} \sqrt[n]{\left|f_{n}\right|}=1 .
$$

We say that a function $\varphi$ interpolates the coefficients of the series (1), if

$$
\varphi(n)=f_{n} \text { for all } n \in \mathbb{N} .
$$

Recall (see, e.g. [2]) that the indicator function $h_{\varphi}(\theta)$ for an entire function $\varphi$ is defined as the upper limit

$$
h_{\varphi}(\theta)=\varlimsup_{r \rightarrow \infty} \frac{\ln \left|\varphi\left(r e^{i \theta}\right)\right|}{r}, \quad \theta \in \mathbb{R} .
$$

Let $\Delta_{\sigma}$ be the sector $\left\{z=r e^{i \theta} \in \mathbb{C}:|\theta| \leqslant \sigma\right\}, \sigma \in[0, \pi)$. By $\gamma_{\sigma, \rho}$ we denote the open arc $\partial D_{\rho} \backslash \Delta_{\sigma}$.

\footnotetext{
*Alex0708@bk.ru

(c) Siberian Federal University. All rights reserved
} 
There are at least three types of questions of analytic continuation of (1) across the arc $\gamma_{\sigma}$. The first one asks about the conditions for continuation to the whole complex plane except $\partial D_{1} \backslash \Delta_{\sigma}$. The answer is given by Polya's theorem.

Theorem (Polya [3]). The series (1) extends analytically to $\mathbb{C}$, possibly except the arc $\partial D_{1} \backslash \gamma_{\sigma}$, if and only if there exists an entire function of exponential type $\varphi(\zeta)$ interpolating the coefficients $f_{n}$ such that

$$
h_{\varphi}(\theta) \leqslant \sigma|\sin \theta| \text { for }|\theta| \leqslant \pi .
$$

Two other questions concern continuation to the sector $\mathbb{C} \backslash \Delta_{\sigma}$ defined by the arc $\gamma_{\sigma}=$ $\partial D_{1} \backslash \Delta_{\sigma}$, or to a neighbourhood of this arc. Both of them are answered by Arakelian's theorems.

Theorem (Arakelian $[4,5])$. The sum of the series (1) extends analytically to the sector $\mathbb{C} \backslash \Delta_{\sigma}$ if and only if there is an entire function $\varphi$ of exponential type interpolating the coefficients of the series $f_{n}$ whose indicator function $h_{\varphi}(\theta)$ satisfies the condition

$$
h_{\varphi}(\theta) \leqslant \sigma|\sin \theta| \text { for }|\theta|<\frac{\pi}{2} .
$$

The continuation property of $f(z)$ to a neighbourhood of the arc $\gamma_{\sigma}$ was studied in [6] (see also [7]). In this case we refer to $\gamma_{\sigma}$ as an arc of regularity for the series (1).

Theorem (Arakelian [7]). The open arc $\gamma_{\sigma}=\mathbb{C} \backslash \Delta_{\sigma}$ is an arc of regularity of the series (1) if and only if there is an entire function $\varphi$ of exponential type interpolating the coefficients of the series $f_{n}$ whose indicator function $h_{\varphi}(\theta)$ satisfies the conditions: $h_{\varphi}(0)=0$ and

$$
\varlimsup_{\theta \rightarrow 0} \frac{h_{\varphi}(\theta)}{|\theta|} \leqslant \sigma .
$$

The inequality (4) implies (5), and (5) together with (2) and (3) gives $h_{\varphi}(0)=0$.

Sometimes it can be easier to interpolate coefficients by meromorphic functions instead of entire ones. Here we consider interpolating functions of the form

$$
\psi(\zeta)=\phi(\zeta) \frac{\prod_{j=1}^{p} \Gamma\left(a_{j} \zeta+b_{j}\right)}{\prod_{k=1}^{q} \Gamma\left(c_{k} \zeta+d_{k}\right)},
$$

where $\phi(\zeta)$ is entire, $a_{j} \geqslant 0, j=1, \ldots, p$, and

$$
\sum_{j=1}^{p} a_{j}=\sum_{k=1}^{q} c_{k}
$$

Denote also

$$
l=\sum_{k=1}^{q}\left|c_{k}\right|-\sum_{j=1}^{p} a_{j} .
$$

In this paper we find the conditions on a meromorphic interpolating function such that the conclusions of all theorems formulated above still hold.

Theorem 1. The sum of the series (1) extends analytically to $\mathbb{C} \backslash\left(\partial D_{1} \cap \Delta_{\sigma}\right)$ if there exists a meromorphic function $\psi(\zeta)$ of the form (6) interpolating the coefficient $f_{n}$ such that the entire function

satisfies

$$
\varphi(\zeta):=\phi(\zeta) \frac{\prod_{j=1}^{p} a_{j} a_{j} \zeta}{\prod_{k=1}^{q}\left|c_{k}\right|^{c_{k} \zeta}}
$$

$$
h_{\varphi}(\theta)+\frac{\pi}{2} l|\sin \theta| \leqslant \sigma|\sin \theta| \quad \text { for } \quad|\theta| \leqslant \pi
$$


Theorem 2. The sum of the series (1) extends analytically to the open sector $\mathbb{C} \backslash \Delta_{\sigma}$ if there exists a meromorphic function $\psi(\zeta)$ of the form (6) interpolating the coefficients $f_{n}$ such that the entire function

$$
\varphi(\zeta):=\phi(\zeta) \frac{\prod_{j=1}^{p} a_{j} a_{j} \zeta}{\prod_{k+1}^{q}\left|c_{k}\right|^{c_{k} \zeta}}
$$

satisfies the conditions

$$
\text { 1) } \left.h_{\varphi}(0)=0, \quad 2\right) \max \left\{h_{\varphi}\left(-\frac{\pi}{2}\right)+\frac{\pi}{2} l, h_{\varphi}\left(\frac{\pi}{2}\right)+\frac{\pi}{2} l\right\} \leqslant \sigma
$$

Theorem 3. The open arc $\gamma_{\sigma}=\partial D_{1} \backslash \Delta_{\sigma}$ is an arc of regularity for the series (1) if there exists a meromorphic function $\psi(\zeta)$ of the form (6) interpolating the coefficients $f_{n}$ such that the entire function

$$
\varphi(\zeta):=\phi(\zeta) \frac{\prod_{j=1}^{p} a_{j} a_{j} \zeta}{\prod_{k+1}^{q}\left|c_{k}\right|^{c_{k} \zeta}}
$$

satisfies the conditions

$$
\text { 1) } h_{\varphi}(0)=0, \quad \text { 2) } \varlimsup_{\theta \rightarrow 0} \frac{h_{\varphi}(\theta)}{|\theta|}+\frac{\pi}{2} l \leqslant \sigma
$$

\section{Proof of Theorem 2}

To begin with, we prove theorem 2 in the case when all $c_{k}$ are positive, i.e. $l=0$. Then the statement is the following.

The sum of the series (1) extends analytically to the open sector $\mathbb{C} \backslash \Delta_{\sigma}$ if there exists a meromorphic function $\psi(\zeta)$ of the form (6) interpolating the coefficients $f_{n}$ such that the indicator function of

$$
\varphi(\zeta):=\phi(\zeta) \frac{\prod_{j=1}^{p} a_{j} a_{j} \zeta}{\prod_{k+1}^{q} c_{k} c_{k} \zeta}
$$

satisfies the conditions

$$
\text { 1) } h_{\varphi}(0)=0, \quad \text { 2) } \max \left\{h_{\varphi}\left(-\frac{\pi}{2}\right), h_{\varphi}\left(\frac{\pi}{2}\right)\right\} \leqslant \sigma .
$$

The indicator of an entire function of exponential type has the following property [7]: if $h_{\varphi}(0)=0$ then for $\alpha \in(0, \pi)$

$$
\begin{gathered}
h_{\varphi}(\theta) \leqslant c_{\alpha}|\sin \theta| \text { for all }|\theta| \leqslant \alpha, \\
c_{\alpha}=\frac{1}{\sin \alpha} \max \left\{h_{\varphi}(\alpha), h_{\varphi}(-\alpha)\right\} .
\end{gathered}
$$

Let $\varphi$ be an entire function of the form (8) satisfying the conditions (9). Show that the series (1) extends to the open sector $\mathbb{C} \backslash \Delta_{\sigma}$. It follows from the definition of an indicator that

$$
\left|\varphi\left(r e^{i \theta}\right)\right| \leqslant e^{h_{\varphi}(\theta) r+o(r)} \text { for } \theta \in \mathbb{R},
$$

where $o(r)$ is infinitesimally small compared to $r$ as $r \rightarrow \infty$.

Taking into account the property of indicator function stated above, we get

$$
\left|\varphi\left(r e^{i \theta}\right)\right| \leqslant e^{\sigma|\sin \theta| r+o(r)} \text { for }|\theta| \leqslant \frac{\pi}{2} .
$$


Since $\varphi(\zeta)$ has the form (8), we obtain the inequality

$$
\left|\phi\left(r e^{i \theta}\right)\right| \frac{\prod_{j=1}^{p}\left|a_{j}^{a_{j} r e^{i \theta}}\right|}{\prod_{k=1}^{q}\left|c_{k}^{c_{k} r e^{i \theta}}\right|} \leqslant e^{\sigma|\sin \theta| r+o(r)} \text { for }|\theta| \leqslant \frac{\pi}{2},
$$

which in terms of $\zeta=\xi+i \eta$ is written as

$$
|\phi(\zeta)| \leqslant\left(\frac{\prod_{j=1}^{p}\left|a_{j}^{a_{j} \zeta}\right|}{\prod_{k=1}^{q}\left|c_{k}^{c_{k} \zeta}\right|}\right)^{-1} e^{\sigma|\eta|+o(|\zeta|)} \text { for } \zeta \in \Delta_{\frac{\pi}{2}}
$$

We need the following estimate.

Lemma 1. For all $\zeta \in \Delta_{\frac{\pi}{2}}$

$$
\left|\frac{\prod_{j=1}^{p} \Gamma\left(a_{j} \zeta+b_{j}\right)}{\prod_{k=1}^{q} \Gamma\left(c_{k} \zeta+d_{k}\right)}\right| \leqslant \frac{\prod_{j=1}^{p}\left|a_{j}^{a_{j} \zeta}\right|}{\prod_{k=1}^{q}\left|c_{k}^{c_{k} \zeta}\right|} e^{o(|\zeta|)} .
$$

Proof. It is easy to see that for $|\zeta| \rightarrow \infty$ one has

$$
|a \zeta|^{a \xi}\left(1-\frac{|b|}{|a \zeta|}\right)^{|a \zeta|} e^{-a \eta \arg (\zeta)} \leqslant|a \zeta+b|^{a \zeta} \leqslant|a \zeta|^{a \xi}\left(1+\frac{|b|}{|a \zeta|}\right)^{|a \zeta|} e^{-a \eta \arg (\zeta)}
$$

This fact together with Stirling's formula gives

$$
\begin{aligned}
& \frac{\prod_{j=1}^{p}\left|\Gamma\left(a_{j} \zeta+b_{j}\right)\right|}{\prod_{k=1}^{q}\left|\Gamma\left(c_{k} \zeta+d_{k}\right)\right|} \sim \frac{\prod_{j=1}^{p}\left|\left(a_{j} \zeta+b_{j}\right)^{\left(a_{j} \zeta+b_{j}\right)} e^{-\left(a_{j} \zeta+b_{j}\right)}\left(2 \pi\left(a_{j} \zeta+b_{j}\right)\right)^{\frac{1}{2}}\right|}{\prod_{k=1}^{q}\left|\left(c_{k} \zeta+d_{k}\right)^{\left(c_{k} \zeta+d_{k}\right)} e^{-\left(c_{k} \zeta+d_{k}\right)}\left(2 \pi\left(c_{k} \zeta+d_{k}\right)\right)^{\frac{1}{2}}\right|} \leqslant \\
& \leqslant \frac{\prod_{j=1}^{p}\left|a_{j} \zeta\right|^{a_{j} \xi}\left(1+\frac{\left|b_{j}\right|}{\left|a_{j} \zeta\right|}\right)^{\left|a_{j} \zeta\right|} e^{-a_{j} \eta \arg (\zeta)}\left|\left(a_{j} \zeta+b_{j}\right)^{b_{j}} e^{-\left(a_{j} \zeta+b_{j}\right)}\left(2 \pi\left(a_{j} \zeta+b_{j}\right)\right)^{\frac{1}{2}}\right|}{\prod_{k=1}^{q}\left|c_{k} \zeta\right|^{c_{k} \xi}\left(1-\frac{\left|d_{k}\right|}{\left|c_{k} \zeta\right|}\right)^{c_{k} \zeta} e^{-c_{k} \eta \arg (\zeta)}\left|\left(c_{k} \zeta+d_{k}\right)^{d_{k}} e^{-\left(c_{k} \zeta+d_{k}\right)}\left(2 \pi\left(c_{k} \zeta+d_{k}\right)\right)^{\frac{1}{2}}\right|} \leqslant \\
& \leqslant \frac{\prod_{j=1}^{p}\left|a_{j}^{a_{j} \zeta}\right|}{\prod_{k=1}^{q}\left|c_{k}^{c_{k} \zeta}\right|}\left|\zeta^{\zeta\left(\sum_{j=1}^{p} a_{j}-\sum_{k=1}^{q} c_{k}\right)}\right|\left|e^{-\zeta\left(\sum_{j=1}^{p} a_{j}-\sum_{k=1}^{q} c_{k}\right)}\right| \times \\
& \times \frac{\prod_{j=1}^{p}\left(1+\frac{\left|b_{j}\right|}{\left|a_{j} \zeta\right|}\right)^{a_{j} \xi} e^{-a_{j} \eta \arg (\zeta)}}{\prod_{k=1}^{q}\left(1+\frac{\left|d_{k}\right|}{\left|c_{k} \zeta\right|}\right)^{c_{k} \xi} e^{-c_{k} \eta \arg (\zeta)}} \times \frac{\prod_{j=1}^{p}\left|a_{j} \zeta+b_{j}\right|^{b_{j}} e^{-b_{j}}\left|2 \pi\left(a_{j} \zeta+b_{j}\right)\right|^{\frac{1}{2}}}{\prod_{k=1}^{q}\left|c_{k} \zeta+d_{k}\right|^{d_{k}} e^{-d_{k}} \mid 2 \pi\left(c_{k} \zeta+d_{k}\right)^{\frac{1}{2}}} .
\end{aligned}
$$

In view of (7), this inequality after some simplifications turns into

$$
\left|\frac{\prod_{j=1}^{p} \Gamma\left(a_{j} \zeta+b_{j}\right)}{\prod_{k=1}^{q} \Gamma\left(c_{k} \zeta+d_{k}\right)}\right| \leqslant \frac{\prod_{j=1}^{p}\left|a_{j}^{a_{j} \zeta}\right|}{\prod_{k=1}^{q}\left|c_{k}^{c_{k} \zeta}\right|}|A \zeta+B|^{C}
$$

where $A, B$ and $C$ are some constants.

Since $|A \zeta+B|^{C}=e^{\ln |A \zeta+B|^{C}}$ and

$$
\lim _{|\zeta| \rightarrow \infty} \frac{\ln |A \zeta+B|^{C}}{|\zeta|}=0
$$

we get $|A \zeta+B|^{C}=e^{o(|\zeta|)}$ as $\zeta \rightarrow \infty$, i.e. the lemma's statement.

It follows form (10) and (11) that for a meromorphic function $\psi(\zeta)$ defined by $(6)$ we have 


$$
|\psi(\zeta)| \leqslant e^{\sigma|\eta|+o(|\zeta|)} \text { for } \zeta \in \Delta_{\frac{\pi}{2}}
$$

Consider the following function

$$
g(\zeta, z)=\frac{z^{\zeta}}{e^{2 \pi i \zeta}-1}
$$

of two complex variables $\zeta=\xi+i \eta, z=x+i y$. It is meromorphic in $\zeta \in \mathbb{C}$ and holomorphic in $z \in \mathbb{C} \backslash \mathbb{R}_{+}$.

Denote $D^{*}:=\cup_{m \in \mathbb{Z}} D_{1 / 4}(m)$.

Notice that there exists a constant $c>0$ such that

$$
\left|e^{2 \pi i \zeta}-1\right|>\frac{e^{\pi(|\eta|-\eta)}}{c} \text { for } \zeta \in \mathbb{C} \backslash D^{*} .
$$

From this we get the estimate

$$
|g(\zeta, z)|<c e^{\xi \log |z|-(\pi-|\pi-\arg z|)|\eta|}
$$

for $\zeta \in \mathbb{C} \backslash D^{*}$ and $z \in \mathbb{C} \backslash \mathbb{R}_{+}$. Using (12) for $\zeta \in \Delta_{\frac{\pi}{2}} \backslash D^{*}$ and $z \in \mathbb{C} \backslash \mathbb{R}_{+}$, we see that

$$
|\psi(\zeta)||g(\zeta, z)|<c e^{\xi \log |z|-(\pi-\sigma-|\pi-\arg z|)|\eta|+o(|\zeta|)} .
$$

For $\zeta \in\left(\Delta_{\frac{\pi}{2}} \backslash D^{*}\right)$ and $z \in \mathbb{C} \backslash \Delta_{\sigma+\delta}$ there is the following bound

$$
|\psi(\zeta)||g(\zeta, z)|<c e^{\xi \log |z|-\delta|\eta|+o(|\zeta|)} .
$$

Consider the integral

$$
I_{m}=\int_{\partial G_{m}} \psi(\zeta) g(\zeta, z) d \zeta
$$

over the oriented boundary of $G_{m}$ that consists of the segments (see Fig. 1)

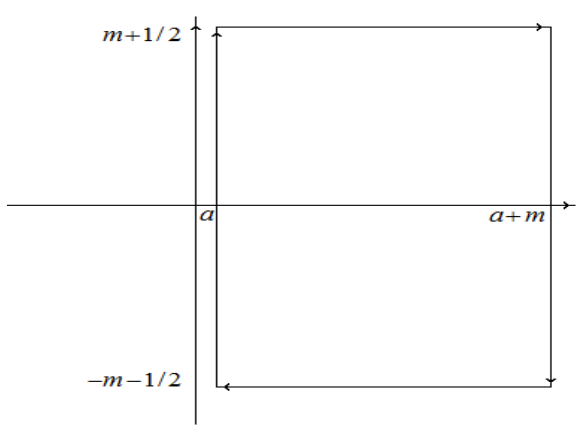

Fig. 1. 


$$
\begin{aligned}
\Gamma_{m}^{1} & =\left[a-i\left(m+\frac{1}{2}\right), a+i\left(m+\frac{1}{2}\right)\right], \\
\Gamma_{m}^{2} & =\left[a+i\left(m+\frac{1}{2}\right), a+m+i\left(m+\frac{1}{2}\right)\right], \\
\Gamma_{m}^{3} & =\left[a+m+i\left(m+\frac{1}{2}\right), a+m-i\left(m+\frac{1}{2}\right)\right], \\
\Gamma_{m}^{4} & =\left[a+m-i\left(m+\frac{1}{2}\right), a-i\left(m+\frac{1}{2}\right)\right]
\end{aligned}
$$

where $\frac{1}{4}<a<\frac{3}{4}$.

The integral $I_{m}$ is the sum of four integrals $I_{m}^{1}, I_{m}^{2}, I_{m}^{3}, I_{m}^{4}$ over $\Gamma_{m}^{1}, \Gamma_{m}^{2}, \Gamma_{m}^{3}, \Gamma_{m}^{4}$ respectively. For $\zeta \in \Delta_{\frac{\pi}{2}} \backslash D^{*}$ и $z \in \mathbb{C} \backslash \Delta_{\sigma+\delta}$ there hold the following estimates

$$
\begin{aligned}
I_{m}^{2} & =\int_{\Gamma_{m}^{2}}|\psi(\zeta) g(\zeta, z)||d \zeta| \leqslant c e^{-\delta\left(m+\frac{1}{2}\right)} \int_{a}^{a+m} e^{\xi \ln |z|+o(|\zeta|)} d \xi, \\
I_{m}^{3} & =\int_{\Gamma_{m}^{3}}|\psi(\zeta) g(\zeta, z)||d \zeta| \leqslant c e^{(a+m) \ln |z|+o(m)} \int_{-i\left(m+\frac{1}{2}\right)}^{i\left(m+\frac{1}{2}\right)} d \eta, \\
I_{m}^{4} & =\int_{\Gamma_{m}^{4}}|\psi(\zeta) g(\zeta, z)||d \zeta| \leqslant c e^{-\delta\left(m+\frac{1}{2}\right)} \int_{a+m}^{a} e^{\xi \ln |z|+o(|\zeta|)} d \xi .
\end{aligned}
$$

We see that for $z \in D_{1} \backslash \Delta_{\sigma+\delta}$ the integrals $I_{m}^{2}, I_{m}^{3}, I_{m}^{4}$ tend to 0 as $m \rightarrow \infty$.

Thus,

$$
\lim _{m \rightarrow \infty} I_{m}=\lim _{m \rightarrow \infty} \int_{\partial G_{m}} \psi(\zeta) g(\zeta, z) d \zeta=\lim _{m \rightarrow \infty} \int_{\Gamma_{m}^{1}} \psi(\zeta) g(\zeta, z) d \zeta=\lim _{m \rightarrow \infty} I_{m}^{1} .
$$

In the domain $G_{m}$, the integrand has simple poles in real integer points and finitely many poles in points $\frac{-\nu-b_{j}}{a_{j}} \in G_{m} \quad \nu=0,1, \ldots$ (recall that $a_{j}, b_{j}$ are parameters in the definition (6) of $\psi(\zeta))$.

The residue theorem yields

$$
\int_{\partial G_{m}} \varphi(\zeta) g(\zeta, z) d \zeta=\sum_{n=1}^{m} \varphi(n) z^{n}+P(z),
$$

where $P(z)$ is a polynomial.

Consider the integral

$$
I=\int_{a-i \infty}^{a+i \infty} \varphi(\zeta) g(\zeta, z) d \zeta
$$


For $\zeta=a+i \eta$ and $z \in \mathbb{C} \backslash \Delta_{\sigma+\delta}$ we have

$$
|\varphi(\zeta)||g(\zeta, z)|<c e^{a \ln |z|-\delta|\eta|+o(|\zeta|)} .
$$

It follows from this inequality that the integral $I$ converges absolutely and uniformly on any compact subset $K \subset \mathbb{C} \backslash \Delta_{\sigma+\delta}$, and defines a holomorphic function on the set of interior points of $K$. For $z \in D_{1} \backslash \Delta_{\sigma+\delta}$

$$
\int_{\Gamma_{m}^{1}} \varphi(\zeta) g(\zeta, z) d \zeta \rightarrow \int_{a-i \infty}^{a+i \infty} \varphi(\zeta) g(\zeta, z) d \zeta \text { as } m \rightarrow \infty
$$

Since $I_{m} \rightarrow I$ as $m \rightarrow \infty, I(z)=f(z)+P(z)$ for $z \in D_{1} \cap K^{o}$. This means that $f(z)$ extends analytically to $K^{o}$. Because $K$ is an arbitrary compact set in $\mathbb{C} \backslash \Delta_{\sigma+\delta}$ for any small $\delta$, the function $f(z)$ extends to the open sector $\mathbb{C} \backslash \Delta_{\sigma}$. Thus, the theorem is proved if all $c_{k}$ are positive.

Prove now the theorem in the case when $c_{k}$ may be negative. Without loss of generality we may assume that only $c_{q}$ among $c_{k}$ is negative, i.e. $\frac{l}{2}=-c_{q}$. Then

$$
\begin{gathered}
\psi(\zeta)=\phi(\zeta) \frac{\prod_{j=1}^{p} \Gamma\left(a_{j} \zeta+b_{j}\right)}{\prod_{k=1}^{q-1} \Gamma\left(c_{k} \zeta+d_{k}\right) \Gamma\left(-\frac{l}{2} \zeta+d\right)}, \\
\varphi(\zeta):=\phi(\zeta) \frac{\prod_{j=1}^{p} a_{j}^{a_{j} \zeta}}{\prod_{k+1}^{q}\left|c_{k}\right|^{c_{k} \zeta}}=\phi(\zeta) \frac{\prod_{j=1}^{p} a_{j}{ }^{a_{j} \zeta}\left(\frac{l}{2}\right)^{\frac{l}{2} \zeta}}{\prod_{k+1}^{q-1} c_{k} c_{k} \zeta} .
\end{gathered}
$$

According to the condition of the theorem

$$
\max \left\{h_{\varphi}\left(-\frac{\pi}{2}\right)+\frac{\pi}{2} l, h_{\varphi}\left(\frac{\pi}{2}\right)+\frac{\pi}{2} l\right\} \leqslant \sigma
$$

Note that the function $\psi(\zeta)$ may be rewritten in the form (6) such that all $c_{k}$ are positive

$$
\psi(\zeta)=\phi(\zeta) \frac{\prod_{j=1}^{p} \Gamma\left(a_{j} \zeta+b_{j}\right)}{\prod_{k=1}^{q-1} \Gamma\left(c_{k} \zeta+d_{k}\right)} \Gamma\left(1+\frac{l}{2} \zeta+d\right) \sin \pi\left(-\frac{l}{2} \zeta-d\right) .
$$

Consider now the entire function

$$
\tilde{\varphi}(\zeta):=\phi(\zeta) \sin \pi\left(-\frac{l}{2} \zeta-d\right) \frac{\prod_{j=1}^{p} a_{j}^{a_{j} \zeta}\left(\frac{l}{2}\right)^{\frac{l}{2} \zeta}}{\prod_{k+1}^{q-1} c_{k} c_{k} \zeta}
$$

Its indicator is bounded

$$
h_{\tilde{\varphi}}(\theta)=\lim _{r \rightarrow \infty} \frac{1}{r} \ln \left|\phi\left(r e^{i \theta}\right) \frac{\prod_{j=1}^{p} a_{j}^{a_{j} r e^{i \theta}}\left(\frac{l}{2}\right)^{\frac{l}{2} r e^{i \theta}}}{\prod_{k+1}^{q-1} c_{k} c_{k} r e^{i \theta}} \frac{e^{i \pi \frac{l}{2} r e^{i \theta}}-e^{-i \pi \frac{l}{2} r e^{i \theta}}}{2 i}\right| \leqslant h_{\varphi}(\theta)+\pi \frac{l}{2}|\sin (\theta)| .
$$

Thus

$$
h_{\tilde{\varphi}}(0)=0, \quad h_{\tilde{\varphi}}\left( \pm \frac{\pi}{2}\right) \leqslant \sigma
$$

The function $\tilde{\varphi}(\zeta)$ satisfies the conditions of (9), hence the sum of the series (1) extends analytically to the open sector $\mathbb{C} \backslash \Delta_{\sigma}$. Theorem 2 is proof. 
The proof of Theorem 3 is largely similar to that of Theorem 2. Namely, from condition 2) of Theorem 3 it follows that for any $\alpha>0$ there exists $\delta>0$ such that $h_{\varphi}(\theta) \leqslant(\sigma+\delta)|\sin \theta|$ for $|\theta| \leqslant \alpha$. Consequently, the bounds (12) and (13) for the modulus of $\psi(\zeta)$ and $\psi(\zeta) g(\zeta, z)$ hold for $\zeta \in \Delta_{\alpha}$. The domains $G$ and $G_{m}$ become

$$
G=D_{1} \cup \Delta_{\alpha}^{o} \text { and } G_{m}=\left\{\zeta=\xi+i \eta \in G: \xi \leqslant m+\frac{1}{2}\right\}
$$

(see Fig. 2), i.e. $\partial G_{m}=\Gamma_{m}^{1} \cup \Gamma_{m}^{2}$.

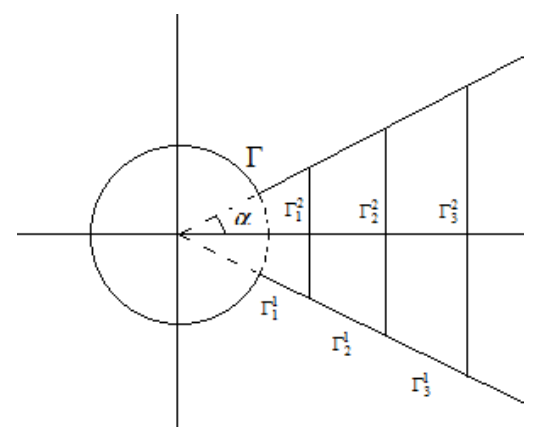

Fig. 2.

The integral $I_{m}$ is then the sum $I_{m}^{1}$ and $I_{m}^{2}$ over $\Gamma_{m}^{1}$, and $\Gamma_{m}^{2}$, and for $z \in K \cap D_{1}^{o}$ the integral $I_{m}^{2} \rightarrow 0$ as $m \rightarrow \infty$.

The integral $I$ over $\partial G$ converges for $\zeta \in \Delta_{\alpha}, z \in K$, (Fig. 3) where $K=D_{e^{\varepsilon}} \backslash\left(\Delta_{\sigma+2 \delta}^{o} \cup D_{\frac{1}{2}}\right)$, $\varepsilon=\frac{\delta \sin \alpha}{2}$

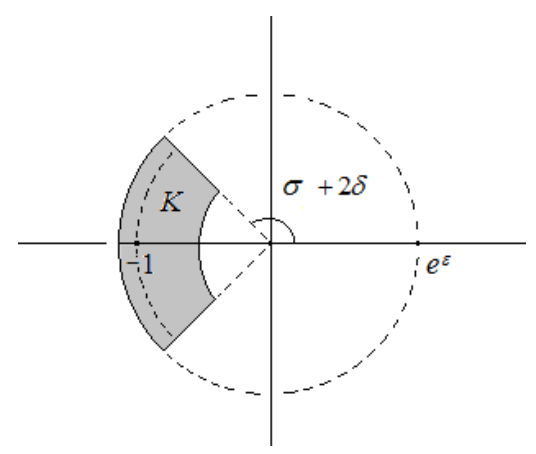

Fig. 3.

The rest of the proof is the same.

As for the proof Theorem 1, it is enough to note that the main estimates (12) and (13) hold for all $\zeta \in \mathbb{C}$. Therfore, by choosing appropriate contours of integrations we prove analytic continuation of the sum of the series to $\mathbb{C} \backslash\left(\partial D_{1} \cap \Delta_{\sigma}\right)$. 


\section{Examples}

Consider two examples clarifying why interpolation of the coefficients by meromorphic functions, and not entire, may be advantageous.

Example 1. Consider the series

$$
f(z)=\sum_{n=0}^{\infty} \frac{\Gamma\left(\frac{2}{3} n+\frac{1}{3}\right) 3^{n}}{\Gamma(n+1) \Gamma\left(-\frac{1}{3} n+\frac{4}{3}\right) 2^{\frac{2}{3} n}} z^{n},
$$

whose domain of convergence is the unit disk. Its coefficients

$$
f_{n}=\frac{\Gamma\left(\frac{2}{3} n+\frac{1}{3}\right) 3^{n}}{\Gamma(n+1) \Gamma\left(-\frac{1}{3} n+\frac{4}{3}\right) 2^{\frac{2}{3} n}}
$$

are given by the values of a meromorphic function of the form (6), namely,

$$
\psi(\zeta)=\frac{3^{\zeta}}{2^{\frac{2}{3} \zeta}} \frac{\Gamma\left(\frac{2}{3} \zeta+\frac{1}{3}\right)}{\Gamma(\zeta+1) \Gamma\left(-\frac{1}{3} \zeta+\frac{4}{3}\right)} .
$$

In this case the entire function from Theorem 2 is

$$
\varphi(\zeta)=\frac{3^{\zeta}}{2^{\frac{2}{3} \zeta}} \frac{\left(\frac{2}{3}\right)^{\frac{2 \zeta}{3}}}{\left(\frac{1}{3}\right)^{\frac{-\zeta}{3}}} \equiv 1 .
$$

Here $l=1+\frac{1}{3}-\frac{2}{3}=\frac{2}{3}, \quad h_{\varphi}(\theta)=0$ and $\max \left\{h_{\varphi}\left(-\frac{\pi}{2}\right)-\frac{\pi}{3}, h_{\varphi}\left(\frac{\pi}{2}\right)+\frac{\pi}{3}\right\} \leqslant \frac{\pi}{3}$.

According to Theorem 2, the series (14) extends analytically to the open sector $\mathbb{C} \backslash \Delta_{\frac{\pi}{3}}$.

Note that the series (17) is the normalized series representing a branch of solution to the algebraic equation $y^{3}-z y-1=0$. This branch has poles in $e^{-i \frac{2}{3} \pi}$ and $e^{i \frac{2}{3} \pi}$ and extends to the sector $\mathbb{C} \backslash \Delta_{\frac{2}{3} \pi}[9]$.

It seems that an entire function interpolating the coefficients cannot be constructed so easily.

Example 2. Consider now the series

$$
f(z)=\sum_{n=0}^{\infty} \frac{\Gamma\left(\frac{n}{3}+\frac{1}{3}\right) 3^{n}}{\Gamma(n+1) \Gamma\left(\frac{-2 n}{3}+\frac{4}{3}\right) 2^{\frac{2 n}{3}}} z^{n},
$$

with the same domain of convergence the unit disk. Its coefficients are

$$
f_{n}=\frac{\Gamma\left(\frac{n}{3}+\frac{1}{3}\right) 3^{n}}{\Gamma(n+1) \Gamma\left(\frac{-2 n}{3}+\frac{4}{3}\right) 2^{\frac{2 n}{3}}} .
$$

They are interpolated by the following entire function

$$
\varphi(z)=\frac{2 \pi}{3^{\frac{1}{2}}} \frac{2^{-\frac{2}{3} z}}{\Gamma\left(\frac{z}{3}+\frac{2}{3}\right) \Gamma\left(\frac{z}{3}+1\right) \Gamma\left(\frac{4}{3}-\frac{2 z}{3}\right)} .
$$

Indeed, in Gauss's multiplication formula

$$
\begin{gathered}
\Gamma(w) \Gamma\left(w+\frac{1}{m}\right) \ldots \Gamma\left(w+\frac{m-1}{m}\right)=m^{\frac{1}{2}-m w}(2 \pi)^{\frac{m-1}{2}} \Gamma(m w) \\
-181-
\end{gathered}
$$


let $m=3, w=\frac{n}{3}+\frac{1}{3}$, then

$$
\Gamma\left(\frac{n}{3}+\frac{1}{3}\right) \Gamma\left(\frac{n}{3}+\frac{2}{3}\right) \Gamma\left(\frac{n}{3}+1\right)=3^{-\frac{1}{2}-n} 2 \pi \Gamma(n+1) .
$$

Express $\Gamma\left(\frac{n}{3}+\frac{1}{3}\right)$ through the other terms of this identity and substitute it into the expression for $f_{n}$, to see that $\varphi(n)=f_{n} n \in \mathbb{N}$.

Estimate $|\varphi(r)|$ by using Stirling's formula

$$
\begin{gathered}
|\varphi(r)|=\left|\frac{2}{3^{\frac{1}{2}}} \frac{2^{\frac{2}{3} r} \Gamma\left(\frac{2 r}{3}-\frac{1}{3}\right) \sin \left(\pi \frac{2 r-1}{3}\right)}{\Gamma\left(\frac{r}{3}+\frac{2}{3}\right) \Gamma\left(\frac{r}{3}+1\right)}\right| \sim \\
\sim \frac{2}{3^{\frac{1}{2}}} \frac{2^{\frac{2}{3} r}\left(2 \pi \frac{2 r-1}{3}\right)^{\frac{1}{2}}\left(\frac{2 r}{3}-\frac{1}{3}\right)^{\frac{2 r}{3}-\frac{1}{3}} e^{-\left(\frac{2 r}{3}-\frac{1}{3}\right)}}{\left(2 \pi \frac{r+2}{3}\right)^{\frac{1}{2}}\left(\frac{r}{3}+\frac{2}{3}\right)^{\frac{r}{3}+\frac{2}{3}} e^{-\left(\frac{r}{3}+\frac{2}{3}\right)}} \frac{\sin \left(\pi \frac{2 r-1}{3}\right)}{\left(2 \pi\left(\frac{r}{3}+1\right)\right)^{\frac{1}{2}}\left(\frac{r}{3}+1\right)^{\frac{r}{3}+1} e^{-\left(\frac{r}{3}+1\right)}} \leqslant C r+e^{o(r) .}
\end{gathered}
$$

It follows that

$$
h_{\varphi}(0)=\varlimsup_{r \rightarrow \infty} \frac{\ln |\varphi(r)|}{r} \leqslant \varlimsup_{r \rightarrow \infty} \frac{\ln \left(C r+e^{o(r)}\right)}{r} \leqslant 0,
$$

on the other hand

$$
h_{\varphi}(0) \geqslant \varlimsup_{n \rightarrow \infty} \frac{\ln |\varphi(n)|}{n}=\varlimsup_{n \rightarrow \infty} \ln \left|f_{n}\right|^{\frac{1}{n}}=0,
$$

therefore $h_{\varphi}(0)=0$.

In order to estimate $\left|\varphi\left(r e^{i \frac{\pi}{2}}\right)\right|$ and $\left|\varphi\left(r e^{-i \frac{\pi}{2}}\right)\right|$ we use the double-sided estimate for the Gamma-function (see [8])

$$
c_{1}(|y|+1)^{x-\frac{1}{2}} e^{-\frac{\pi}{2}|y|} \leqslant \Gamma(x+i y) \leqslant c_{2}(|y|+1)^{x-\frac{1}{2}} e^{-\frac{\pi}{2}|y|},
$$

where $x \in K \subset \mathbb{R} \backslash\{0,-1,-2, \ldots\}, K$ is compact. The constants $c_{1}$ and $c_{2}$ depend on the choice of $K, y \in \mathbb{R}$. Then

$$
\left|\varphi\left(r e^{ \pm i \frac{\pi}{2}}\right)\right| \leqslant C \frac{e^{\frac{\pi}{6} r} e^{\frac{\pi}{6} r} e^{\frac{2 \pi}{6} r}}{c_{1}\left(\frac{r}{3}+1\right)^{\frac{2}{3}-\frac{1}{2}} c_{1}\left(\frac{r}{3}+1\right)^{1-\frac{1}{2}} c_{1}\left(\frac{2 r}{3}+1\right)^{\frac{4}{3}-\frac{1}{2}}}
$$

or

$$
\ln \left|\varphi\left(r e^{ \pm i \frac{\pi}{2}}\right)\right| \leqslant \frac{2 \pi}{3} r+o(r)
$$

Therefore

$$
h_{\varphi}\left( \pm \frac{\pi}{2}\right) \leqslant \frac{2 \pi}{3} .
$$

It follows from Arakelian's Theorem [4] that the series (17) extends to the open sector $\mathbb{C} \backslash \Delta_{\frac{2}{3} \pi}$.

On the other hand, the coefficients of the series (17) are interpolated by the meromorphic function

$$
\psi(\zeta)=\frac{3^{\zeta}}{2^{\frac{2}{3} \zeta}} \frac{\Gamma\left(\frac{1}{3} \zeta+\frac{1}{3}\right)}{\Gamma(\zeta+1) \Gamma\left(-\frac{2}{3} \zeta+\frac{4}{3}\right)} .
$$

The entire function of Theorem 2 is

$$
\Phi(\zeta)=\frac{3^{\zeta}}{2^{\frac{2}{3} \zeta}} \frac{3^{-\frac{1}{3 \zeta}}}{\left(-\frac{2}{3}\right)^{-\frac{2}{3} \zeta}} \equiv 1,
$$


and $l=1+\frac{2}{3}-\frac{1}{3}=\frac{4}{3}, \quad h_{\varphi}(\theta)=0$ and $\max \left\{h_{\varphi}\left(-\frac{\pi}{2}\right)-\frac{2 \pi}{3}, h_{\varphi}\left(\frac{\pi}{2}\right)+\frac{2 \pi}{3}\right\} \leqslant \frac{2 \pi}{3}$.

Therefore, by Theorem 2 the series (17) extends to the open sector $\mathbb{C} \backslash \Delta_{\frac{2}{3} \pi}$.

The research is supported by the grant of the RF Government for carrying research under supervision of leading scientists in Siberian Federal University (Project 14.Y26.31.0006), the research was also carried out by the partial financial support of the fund "Dynasty".

\section{References}

[1] L.Bieberbach, Analytische Fortsetzung, Springer, Berlin, 1955.

[2] L.I.Ronkin, Introduction to the Theory of Entire Functions of Several Variables, Nauka, Moscow, 1971 (in Russian).

[3] G.Polya, Untersuchungen über Lücken und Singularitäten von Potenzreihen, Mathematische Zeitschrift, 29(1929), 549-640.

[4] N.Arakelian, On efficient analytic continuation of power series, Mathematics of the USSRSbornik, 52(1985), no. 1, 21-39.

[5] N.Arakelian, V.Martirosyan, Power series: Analytic continuation and location of singularities, University Press, Yerevan, 1991 (in Russian).

[6] N.U.Arakelian, Approximation by entire functions and analytic continuation, Progress in Approximation theory (Tampa, FL, 1990), Computational Mathematical Series, Springer, New York, 19(1992), 295-313.

[7] N.Arakelian, W.Luh, J.Muller, On the localization of singularities of lacunar power series, Complex Variables and Elliptic Equations, 52(2007), no. 7, 561-573.

[8] O.N.Zhdanov, A.K.Tsikh, Investigation of multiple Mellin-Barnes integrals by means of multidimensional residues, Sib. Math. J., 39(1998), no. 2, 281-298.

[9] I.A.Antipova, E.N.Mikhalkin, Analytic continuations of a general algebraic function by means of Puiseux series, Proceedings of the Steklov Institute of Mathematics, 279(2012), no. $1,3-13$.

\section{Аналитическое продолжение степенных рядов путем интерполяции коэффициентов мероморфными функциями}

\section{Александр Д. Мкртчян}

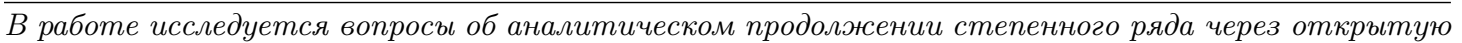
дугу на границе круга сходимости. Ответ на такой вопрос дан в терминах мероморфной функции специального вида, интерполирующей коэффичиенты ряда. Получены условия при которых сумма ряда аналитически продолжается в некоторую окрестность дуги в сектор, определенный дугой, во всю комплексную плоскость, кроме некоторой дуги.
}

Ключевые слова: степенные ряды, аналитическое продолжение, интерполирующая мероморфная функция, индикатор функиия. 